\title{
Do Australian adolescent and young adults' experiences of cancer care influence their quality of life?
}

RUNNING TITLE: AYA experiences of cancer care and quality of life

Gemma Skaczkowski $^{\mathrm{a}, \mathrm{c}, \mathrm{d}}$, Victoria White ${ }^{\mathrm{a}, \mathrm{b} *}$, Kate Thompson ${ }^{\mathrm{e}}$, Helen Bibby ${ }^{\mathrm{a}}$, Michael

Coory $^{\mathrm{m}}$, Ross Pinkerton, ${ }^{\mathrm{o}}$ Lisa M Orme $^{\mathrm{e}, \mathrm{f}}$, Rachel Conyers ${ }^{\mathrm{e}, \mathrm{f,n}}$, Marianne B Phillips ${ }^{\mathrm{j}}$, Michael Osborn $^{\mathrm{k}}$, Rosemary Harrup ${ }^{1}$, Antoinette Anazodo ${ }^{\mathrm{g}, \mathrm{h}, \mathrm{i}}$

${ }^{a}$ Centre for Behavioural Research in Cancer, Cancer Council Victoria, Victoria, Australia

${ }^{\mathrm{b}}$ School of Psychology, Deakin University, Victoria, Australia

${ }^{c}$ School of Psychology and Public Health, La Trobe University, Victoria, Australia

${ }^{\mathrm{d}}$ Olivia Newton-John Cancer Wellness \& Research Centre, Austin Health, Victoria, Australia

${ }^{\mathrm{e}}$ Peter MacCallum Cancer Centre, Victoria, Australia

f Children's Cancer Centre, The Royal Children's Hospital, Victoria, Australia

${ }^{g}$ Kids Cancer Centre, Sydney Children's Hospital, New South Wales, Australia

${ }^{\mathrm{h}}$ Nelune Comprehensive Cancer Centre, Prince of Wales Hospital, New South Wales,

Australia

i School of Women's and Children's Health University of New South Wales, Australia

${ }^{j}$ Princess Margaret Hospital for Children, Western Australia, Australia

${ }^{\mathrm{k}}$ Royal Adelaide Hospital, South Australia, Australia

${ }^{1}$ Royal Hobart Hospital, Tasmania, Australia

${ }^{\mathrm{m}}$ Faculty of Medicine, Dentistry and Health Sciences, University of Melbourne, Victoria, Australia

${ }^{\mathrm{n}}$ Murdoch Childrens Research Institute, Victoria, Australia

${ }^{\circ}$ Children's Health Queensland Hospital and Health Service, Queensland, Australia

\section{Corresponding author:}

This is the author manuscript accepted for publication and has undergone full peer review but has not been through the copyediting, typesetting, pagination and proofreading process, which may lead to differences between this version and the Version of Record. Please cite this article as doi: $10.1002 /$ pon.4625

This article is protected by copyright. All rights reserved. 
Victoria White, Centre for Behavioural Research in Cancer, Cancer Council Victoria, 615 St Kilda Road, Melbourne, Victoria, 3004.

T: +61 395146352 E: Vicki.White@ cancervic.org.au

Word count: 4,466

This article is protected by copyright. All rights reserved. 


\begin{abstract}
Objective: To examine the relationship between the cancer care experiences of adolescents and young adults (AYAs) and their quality of life.
\end{abstract}

Methods: 209 AYAs completed a cross-sectional, self-report survey distributed through the population-based cancer registries in two Australian states (New South Wales and Victoria). Eligible AYAs were diagnosed at 15-24 years old with any cancer (excluding early stage melanoma) and were 3-24 months post-diagnosis. Questions examined whether particular care experiences occurred for the patient at different points in the cancer care pathway, including diagnosis, treatment, inpatient care and at the end of treatment. Quality of life was assessed using the FACT-G.

Results: Positive experiences of care at diagnosis, during treatment, during inpatient stays and when finishing treatment were associated with higher functional, emotional and social wellbeing. However, these associations generally became non-significant when communication and support experiences were included in the model. Inpatient experiences positively influenced emotional well-being over and above the effect of communication and support experiences.

Conclusions: The results suggest that, for most AYAs' quality of life outcomes, positive experiences of age-appropriate communication and emotional support may underpin the effect of positive experiences of care throughout the cancer care pathway. The results support the need for communication and support tailored to an AYA audience, as recognised by recent Australian and international guidelines on the care of AYAs with cancer. 
Keywords: Australia, adolescents and young adults, cancer, experiences of care, oncology, quality of life

\section{Background}

In recent years, the psychosocial health care needs and services available to adolescents and young adults (AYAs) with cancer have become the subject of increasing national and international attention [1-3]. AYAs have unique psychosocial needs that differ from adult or paediatric patients [4], with the AYA developmental period involving key cognitive, psychological and physical milestones. A cancer diagnosis can significantly interfere with the development of autonomy and independence, social functioning, interactions with peers and the formation of intimate relationships that characterise this growth phase [4-6]. At a time when most AYAs are developing a sense of their personal and sexual identity, body image and self-worth, AYAs with cancer face uncertainty about their future, temporary or permanent side-effects of treatment, and are placed in a position of dependence on parents with a loss of control over life [5-7].

AYA cancer patients have reported lower physical and mental well-being than sameaged healthy cohorts and/or general population norms in the US, with those currently receiving treatment showing the lowest well-being $[8,9]$. Other work has shown that quality of life (QoL) of AYA cancer patients is worse than that of cancer patients at other ages [10] and many AYAs report age-specific issues such as difficulty fitting in with peers, impacts on family relationships, concerns about potential infertility, and negative impacts on intimate relationships $[11,12]$. 
Several studies have suggested that AYAs' care experiences and the degree to which they perceive gaps in their care (unmet needs) relate to AYA's psychosocial well-being [13]. For instance, analyses of the AYA HOPE study in the US showed that AYAs' unmet information needs have been associated with increased distress, with another study finding unmet needs were associated with worse QoL in physical and mental domains $[14,15]$. An Australian study[16] found that unmet physical/daily living needs and health system/information needs were key predictors of AYA cancer patients' depression and anxiety, respectively. A recent US study found that AYAs with higher ratings of the negative impact of cancer had worse mental QoL and increased distress, while a positive impact of cancer showed the opposite relationship [17].

Perceived quality of care has been conceptualised as a combination of several dimensions of patient experience, including (1) respect for preferences, (2) care coordination, (3) information, education and communication, (4) physical comfort, (5) emotional support, (6) involvement of family and friends, and (7) continuity and transition [18, 19]. A US study of patient experiences found emotional support played a key role in determining quality perceptions [18]. For AYAs, age-appropriate information and emotional support have been consistently identified as key needs, including health service staff being knowledgeable about AYAs' psychosocial needs and being able to tailor and communicate information to their age and developmental stage [13]. Communication and support experiences throughout the entire cancer journey may have a significant impact on AYAs' well-being.

In a number of studies, AYAs have expressed a desire for opportunities to meet other young cancer patients/survivors [20-22] as well as the importance of support from peers and 
family $[23,24]$. In an attempt to meet these needs, specialist AYA cancer treatment services have been developed in many countries, including Australia. These services aim to provide a treatment environment, emotional support and care that are tailored to the unique psychosocial needs of this cohort $[2,25,26]$ with the expectation that AYAs' care experiences will improve and their unmet needs reduce. These centres also aim to improve the coordination of AYA's care, which has been shown to play an important role in perceived care quality [18]. However, to date, no study has examined whether more positive care experiences (e.g. age-appropriateness, emotional support, greater information provision) are associated with reduced needs and consequently better QoL in AYAs.

In this paper we aim to fill this gap in the literature by first examining the QoL of AYAs who are, on average, within a year of their diagnosis and second, by examining how care experiences are related to AYAs' QoL.

\section{Methods}

The data presented here form part of a larger study of AYA's experiences of cancer care in Australia [27]. This was a cross-sectional, retrospective self-report survey on cancer care experiences. In brief, AYA cancer patients, aged 15-24 years, with any form of cancer (except early stage melanoma ${ }^{1}$ ) were approached about the study by postal mail. Eligible participants were 3-24 months post-diagnosis, had an expected survival of at least 24 months, were physically and mentally able to participate and able to speak English (determined by

\footnotetext{
${ }^{1}$ Early stage melanoma is likely to be treated only with surgery, which may be conducted at a day clinic rather than a hospital, and have better prognoses than later-stage melanomas or other cancers. As such, their experiences of diagnosis and treatment are different from those of other cancer patients.
} 
their treating clinician). Recruitment and case identification was conducted by populationbased cancer registries (CRs) in two Australian states (New South Wales (NSW) and Victoria). In Australia, all cancer cases are required by law to be reported to state-based cancer registries. Recruitment therefore covered the entire population of AYA cancer patients in these two states. Surveys were included in the approach letter to patients and could be completed in hardcopy or online.

CRs initially contacted treating clinicians by letter to confirm eligibility. Unless the clinician indicated otherwise within 4 weeks, CRs mailed eligible patients a study pack containing the survey and information on consent procedures. Participants could provide hardcopy or online consent while for minors (15-17 years), consent from both the patient and their parent/guardian was required. A reminder letter was sent at 2 and 4 weeks.

\section{Measures}

\section{Experiences of care}

Experience of care (EOC) questions asked whether specific events/experiences occurred for patients at various stages throughout their cancer care. Questions were based on the Critical Care Events Survey [28] and adapted to address AYA-specific issues as identified in the literature $[5,13,22,29]$. Qualitative interviews were conducted with health care professionals (HCPs) and AYA cancer patients to further develop items. Questions covered all points in the cancer pathway, from seeking advice prior to diagnosis, to treatment, inpatient care and finishing treatment. Communication and support throughout care was also assessed, with questions assessing information provision, age-appropriateness of services, 
interactions with HCPs, and whether patients felt supported. Responses to most items were made on categorical scales, with response options indicating the degree of certainty that the event/action occurred ('Yes, definitely', 'Yes, I think so', 'No, I don't think so', 'No, definitely not', 'Not sure') or the frequency with which a particular event/action occurred ('Always', 'Sometimes', 'Rarely', 'Never', 'Not sure'). Item responses were dichotomised to indicate 1 'yes, definitely'/'always' versus 0 'other responses' (i.e. optimal care vs. suboptimal care) [30].

To develop scales assessing experiences at each care point, a series of exploratory factor analyses (principal axis factoring with an oblique rotation) were conducted on questionnaire items in each survey section using the tetrachoric correlation matrix (appropriate for binary items). Items were selected where they were conceptually and statistically related to other items on the scale. As items were binary, reliability was assessed through the KuderRichardson Formula 20 (KR-20). Analyses were conducted using STATA v.14 (see Supplementary Materials 1).

Scales reflected experiences of care when AYAs were told they had cancer (Diagnosis; 6 items; KR-20=0.71), the quality and age-appropriateness of information provision and practical/emotional support provided throughout treatment (Communication/Support; 11 items; KR-20=0.88), experiences of inpatient care (Inpatient; 6 items; KR-20=0.87) and experiences at the end of treatment (Finishing treatment; 7 items; KR-20=0.83).

Scales were constructed to reflect experiences of care for patients receiving Surgery ( 8 items; KR-20=0.89), Chemotherapy (7 items; KR-20=0.80) and Radiotherapy (6 items; KR- 
$20=0.81$ ). Items were broadly consistent across the different treatments (e.g. whether patients were given information to help prepare for treatment) although items specific to each treatment were also included (e.g. help managing pain following surgery, managing changes to appearance following chemotherapy).

Quality of life

Physical, social, functional and emotional well-being (PWB, SWB, FWB, EWB respectively) were assessed using the Functional Assessment of Cancer Therapy-General scale (FACT-G)[31]. Participants rated statements about the impact of cancer on their day-today life on a 5-point Likert scale ranging from 'Not at all' to 'Very much'. Scores on the total FACT-G range between 0-108, with higher scores indicating better well-being. Minor adaptations were made to the wording of questions to ensure age-appropriateness, for instance by adding "school/university/technical college" as options in questions assessing impact on “work”. Cronbach's alphas were 0.88 (PWB), 0.83 (SWB), 0.88 (FWB) and 0.75 (EWB).

\section{Demographics and treatment information}

Participants provided information about their age, gender, education level, treatment received and whether treatment was complete. They were also asked to indicate how many hospitals had been involved in their care. Cancer diagnosis and postcode was obtained from the CRs.

\section{Data Analysis}

Development of EOC scales

This article is protected by copyright. All rights reserved. 
Scales were calculated by taking the sum of the number of 'optimal' responses divided by the number of items the participant answered for that scale. Scores on each scale ranged from $0-1$, with higher scores indicating more positive experiences of care. This method was used due to a small amount of missing data ( $<5 \%$ within each subscale) and the fact that some questions were only relevant to some participants and were therefore 'missing' for the remaining participants. Scale scores were calculated if participants provided valid responses for at least $75 \%$ of items on a scale. For the Chemotherapy scale, this was lowered to $50 \%$ since some items only applied to outpatients and were therefore consistently missing for all inpatients. The Surgery, Radiotherapy and Chemotherapy scales correlated well ( $r$ 's $>=0.45)$. Due to small sample sizes for the individual scales, an overall Treatment scale was computed as the average of the three subscale scores.

\section{Main analysis}

Quality of life scores were compared to general population norms for 20-39 year-olds in the Australian state of Queensland [32] (population norms for the 15-24 year age group are not available). Sample scores were converted to a t-distribution with a mean of 50 and a standard deviation of 10. A one-sample t-test examined if the sample score significantly differed from the norm mean [33].

The impact of demographic and treatment-related characteristics on QoL was assessed using unadjusted univariable ANOVAs. A series of multiple regressions examined the influence of experiences of care on each QoL domain. Regressions controlled for demographic and treatment-related factors shown to influence QoL in the previous 
analysis[34]. In the first step of the regression models, the EOC scale of interest was entered along with the control variables. The Communication/Support scale was entered in the second step of all regression models. ANOVAs examined the difference in QoL between patients who attended one hospital compared to two or more hospitals, adjusting for demographic and treatment-related factors shown to influence QoL in the previous analysis. Analyses were conducted in SPSS v.20, unless otherwise indicated.

\section{Results}

Of the 715 surveys sent, 209 were returned (including 25 completing the survey online), giving a response rate of $29 \%$. Participant characteristics were generally similar those of the population of all eligible patients, except for a slight over-representation of females. Two participants were excluded due to insufficient data (i.e. not having commenced treatment and no information being available from the CR). As reported in White et al., [35], participants' mean age was 20.36 years $(\mathrm{SD}=2.97)$, with $63.8 \%$ aged $20-24$ years. The majority of participants were female $(57.5 \%)$ and not currently undergoing treatment $(77.8 \%)$. Seventy per cent of the sample had received surgery, $61.4 \%$ had received chemotherapy and 30.9\% had received radiotherapy. Haematological cancers were the most common (42.0\%) and the least common were Central Nervous System (CNS) tumours $(6.3 \%)$. The average time since diagnosis was 8.5 months $(S D=4.13)$. Fifty-seven percent of participants received treatment at one centre, $33.3 \%$ received treatment at two centres and 9.7\% received treatment at three or more centres. The number of treatment centres attended was associated with the type of treatment received $\left(\chi^{2}(2)=11.47, p=.003\right)$, with patients 
receiving only surgery more likely to attend one hospital (73.2\%), compared to patients receiving surgery and chemotherapy and/or radiotherapy (44.9\%).

\section{Quality of life of AYAs with cancer}

Means for each QoL subscale and overall scale are provided in Table 1. AYAs displayed significantly higher SWB $(t(204)=8.25, p<.001)$ and lower PWB $(t(205)=-5.76$, $p<.001)$ and $\operatorname{EWB}(t(204)=-7.92, p<.001)$ than the general population. Overall well-being and FWB did not differ from the general population ( $p$ 's >.05). A number of demographic and treatment-related characteristics influenced QoL (see Supplementary Materials 2), including gender, location of residence, diagnosis, type of treatment, and whether treatment was complete.

Most patients (71.8\%) stayed as a hospital inpatient at some time during their care, with these patients displaying worse PWB, FWB and overall QoL than those having all their treatment as outpatients (Supplementary Materials 2).

\section{Quality of life and experiences of care}

Average subscale scores for EOC at each point in the cancer care pathway are shown in Table 2. Experiences at the end of treatment were poor, at 0.31 on a scale of $0-1$. Experiences at diagnosis and experiences of overall communication and support were moderate (at 0.41 and 0.48 , respectively) and experiences of treatment and inpatient care were better (at 0.63 and 0.62 , respectively). All scales were moderately-to-highly correlated. 
The relationship between QoL and EOC is shown in Table 1. More positive experiences of communication and support were associated with higher well-being in all domains. Experiences when being diagnosed were positively associated with FWB, EWB and SWB and experiences of treatment were positively associated with FWB, EWB and SWB. In both cases, these associations reduced and became non-significant with the inclusion of Communication/Support to the models.

Inpatient experiences were positively associated with QoL across all domains. With the addition of Communication/Support to the model, only the effect of inpatient experiences on EWB remained significant. While end-of-treatment care experiences were positively associated with FWB, EWB and SWB, these associations reduced once Communication/Support was included in the model.

\section{Quality of life and number of hospitals involved in care}

Patients attending only one hospital for their care $(\mathrm{M}(\mathrm{SE})=86.99(1.80))$ displayed better overall well-being than patients attending two or more hospitals $(\mathrm{M}(\mathrm{SE})=81.95(1.79)$, $\mathrm{F}(1,194)=4.81, p=.030)$. FWB was also significantly higher for patients attending only one hospital $(\mathrm{M}(\mathrm{SE})=22.02(0.68))$ than those attending two or more hospitals $(\mathrm{M}(\mathrm{SE})=19.18(0.68), \mathrm{F}(1,194)=10.58, p=.001)$. There was no evidence that the number of hospitals involved in patients' treatment influenced any other QoL domain.

\section{Discussion}


This is the first Australian study to examine whether the QoL of AYAs with cancer is related to the experiences they have during their care. The results suggest that more positive care experiences at critical points in the cancer care pathway between 3-24 months postdiagnosis were associated with higher well-being overall and in each domain of functioning. This is consistent with the AYA literature emerging from other countries [13-15] as well as the literature on adults [36] and children [37, 38].

Our study demonstrated that an important aspect of care is good communication and support, as we found that the impact of experiences at different care points reduced once communication and support experiences were taken into account. In many instances, communication and support was the only aspect of care associated with well-being in multivariable models.

AYAs have cited age-appropriate information and emotional support as important unmet needs [13] and the provision of these services is a key component of both national and international guidelines on AYA treatment [1, 39-42]. In response to the need for ageappropriate cancer services, in 2008 the Australian Government provided funding for the development of specialised youth cancer services. Our results suggest the appropriateness of this development, though future research is needed.

Interestingly, the only scale whose association with emotional well-being did not diminish when Communication/Support was included was the inpatient experiences scale. One potential explanation is that, unlike experiences at other points in the cancer care pathway, inpatient experiences may have more to do with relationships with hospital staff 
than with information provision, though further research is needed to confirm this hypothesis. Inpatients displayed worse physical and functional well-being compared to outpatients, who may have more flexibility to maintain their 'normal' life, interact with their peers and attend work/school. It is also likely that outpatients receive less intensive treatment than inpatients, which may drive the physical well-being differences found here.

Few studies have quantitatively examined the association between AYAs' care experiences and their QoL. Our findings are consistent with previous studies that report AYAs' unmet needs are associated with impaired well-being [14]. Our finding that EOC can have a direct impact on well-being is consistent with a recent review which outlined the types of issues affecting AYAs' QoL, such as the loss of friendships, inadequate management of pain and side-effects from treatment[12], with our EOC measure including many of these issues.

Participants in this study indicated better SWB, but worse PWB and EWB, than the general population. These results are generally consistent with past studies indicating that physical and mental health among AYA cancer patients is worse than their general population counterparts [10], although US data also showed deficits in social functioning that were not replicated here [8]. However, due to a lack of FACT-G population norms for AYAs in Australia, we compared our sample to 20-39 year olds living in a different Australian state (Queensland) and future research should aim to compare AYA patient data to more comparable norms. 
Results also showed that patients who attended only one hospital showed better FWB than patients who attended two or more hospitals for their care. Functional well-being covers the ability to enjoy life, sleep well, to continue with work/studies and find fulfilment in work/studies; factors which may logically be influenced by the efficient coordination of care. Patients attending one hospital may also be more likely to receive less intensive treatment, though a secondary analysis controlling for treatment [results not shown] indicated the same pattern of results. A caveat to this result is that the survey only measured hospitals involved in a patient's treatment and no data was collected on how many hospitals were involved in the diagnostic pathway or whether they attended additional centres for psychosocial care or fertility treatment/counselling.

\section{Clinical implications}

Research has shown that when people are told a cancer diagnosis, conversations tend to be information-focussed and HCPs often miss opportunities to address emotional concerns expressed by patients and their families [43]. Our results similarly identified that the psychosocial needs of AYAs were not consistently being met throughout their cancer care. Ensuring that at each stage of care, communication is age-appropriate, AYAs understand the information they are given, treatment is tailored to their lifestyle and commitments, and emotional support needs are addressed, are some of the ways in which HCPs can improve the care experiences of AYAs and their subsequent well-being. The results support the need for specialised AYA centres. Where this is not possible, collaboration with AYA specialists to provide secondary consultations and training modules focussing on AYA needs and communication skills may assist HCPs who do not have regular exposure to this group. 
Our study suggests that patients' experiences when finishing treatment are particularly sub-optimal (with a score of 0.31 on a scale of $0-1$ ). As poor experiences at this point of care were associated with lower QoL, the results of this study indicate a clear need for improvement in the provision of services at the end of treatment. Finally, where it is possible to structure AYAs' care within the one institution, our results suggest that this may positively benefit well-being.

\section{Limitations}

Though this is the first population-based assessment of AYAs' experiences of cancer care in Australia, the relatively small sample size and low response rate limit generalizability. However our response rate is similar to other studies examining AYA experiences of care or QoL (25\%[44]; 31\%[17]). A recent Australian study recruiting AYAs through hospitals also achieved a response rate under 30\% (26\%[45]). However, recruitment through CRs ensured that the sample was not biased towards patients attending specific treatment centres or AYA clinicians. A wide range of diagnoses were included and the sample was demographically similar to the eligible cohort identified by the registries. Nonetheless, a larger sample including patients from all Australian states and territories is needed. In particular, a larger sample would provide more certainty in the results including corrections for multiple tests. Additionally, there is a need for longitudinal studies to examine causality and reduce retrospective recall biases, and for quality of life measures that have been validated for this age group and that more directly address AYA-specific issues (such as peer relations and sexual identity). Additionally, our findings are restricted to key points in the cancer care 
pathway in the 3-24 months post-diagnosis, and do not examine experiences outside of these, or experiences further into survivorship or at end-of-life.

\section{Conclusions}

Positive experiences of communication and support predicted better QoL in functional, emotional, social and physical domains, indicating the importance of ageappropriate information and emotional support in the care of AYAs with cancer. HCPs need to be aware of the unique needs to this cohort and how best to incorporate age-appropriate communication and support into their care. Further research is needed to determine the factors contributing to the provision of age-appropriate services, including the effectiveness of specialised AYA centres in this.

This article is protected by copyright. All rights reserved. 
Acknowledgements: We would like to thank the participants in this study.

Funding: This work was supported by a National Health and Medical Research Council of Australia project grant 1012250.

Conflicts of interest: None declared.

Ethics Approval: The study was approved by the Human Research Ethics Committees of Cancer Council Victoria (reference HREC 1103) and the NSW Cancer Institute (reference2013/02/443).

This article is protected by copyright. All rights reserved. 


\section{Reference List}

1. Psychosocial management of AYA cancer patients Working Group. Psychosocial management of AYAs diagnosed with cancer: Guidelines for health professionals. Sydney: Cancer Council Australia; 2012 [cited 21/08/2013]; Available from: http://wiki.cancer.org.au/australia/COSA:Psychosocial_management_of_AYA_cance r_patients.

2. Whiteson M. The Teenage Cancer Trust--advocating a model for teenage cancer services. Eur J Cancer. 2003;39:2688-93.

3. Zebrack B, Mathews-Bradshaw B, Siegel S. Quality cancer care for adolescents and young adults: a position statement. J Clin Oncol. 2010;28:4862-7.

4. Morgan S, Davies S, Palmer S, et al. Sex, drugs, and rock 'n' roll: caring for adolescents and young adults with cancer. J Clin Oncol. 2010;28:4825-30.

5. Abrams AN, Hazen EP, Penson RT. Psychosocial issues in adolescents with cancer. Cancer Treat Rev. 2007;33:622-30.

6. Bellizzi KM, Smith A, Schmidt S, et al. Positive and negative psychosocial impact of being diagnosed with cancer as an adolescent or young adult. Cancer. 2012;118:515562.

7. Seitz DC, Besier T, Goldbeck L. Psychosocial interventions for adolescent cancer patients: a systematic review of the literature. Psycho-Oncology. 2009;18(7):683-90.

8. Smith AW, Bellizzi KM, Keegan TH, et al. Health-related quality of life of adolescent and young adult patients with cancer in the United States: the Adolescent and Young Adult Health Outcomes and Patient Experience study. J Clin Oncol. 2013;31:2136-45.

9. Husson O, Zebrack BJ, Block R, et al. Health-related quality of life in adolescent and young adult patients with cancer: A longitudinal study. J Clin Oncol. 2017;35:652-9.

10. Quinn GP, Goncalves V, Sehovic I, et al. Quality of life in adolescent and young adult cancer patients: a systematic review of the literature. Patient Relat Outcome Meas. 2015;6:19-51.

11. Nightingale CL, Quinn GP, Shenkman EA, et al. Health-related quality of life of young adult survivors of childhood cancer: A review of qualitative studies. J Adolesc Young Adult Oncol. 2011;1:124-32.

12. Sodergren SC, Husson O, Robinson J, et al. Systematic review of the health-related quality of life issues facing adolescents and young adults with cancer. Qual Life Res. 2017.

13. Bibby $\mathrm{H}$, White $\mathrm{V}$, Thompson $\mathrm{K}$, et al. What are the unmet needs and care experiences of adolescents and young adults with cancer? A systematic review. J Adolesc Young Adult Oncol. 2017;6:6-30.

14. Smith AW, Parsons HM, Kent EE, et al. Unmet support service needs and health-related quality of life among adolescents and young adults with cancer: The AYA HOPE study. Front Oncol. 2013;3:75.

15. DeRouen MC, Smith AW, Tao L, et al. Cancer-related information needs and cancer's impact on control over life influence health-related quality of life among adolescents and young adults with cancer. Psycho-Oncology. 2015;24:1104-15. 
16. Dyson GJ, Thompson K, Palmer S, et al. The relationship between unmet needs and distress amongst young people with cancer. Support Care Cancer. 2012;20:75-85.

17. Husson O, Zebrack BJ. Perceived impact of cancer among adolescents and young adults: Relationship with health-related quality of life and distress. Psychooncology. 2017;26:1307-15.

18. Rathert C, Williams ES, McCaughey D, et al. Patient perceptions of patient-centred care: empirical test of a theoretical model. Health Expect. 2015;18(2):199-209.

19. Davis K, Schoenbaum SC, Audet AM. A 2020 vision of patient-centered primary care. J Gen Intern Med. 2005;20(10):953-7.

20. Fern LA, Taylor RM, Whelan J, et al. The art of age-appropriate care: Reflecting on a conceptual model of the cancer experience for teenagers and young adults. Cancer Nurs. 2013;36(5):E27-38.

21. Smith $S$, Davies $S$, Wright $D$, et al. The experiences of teenagers and young adults with cancer - Results of 2004 conference survey. Eur J Oncol Nurs. 2007;11:362-8.

22. Gallini A, Hooker L. Young people's and carer's views on the cancer services they receive. Cancer Nursing Practice. 2005;4:27-32.

23. Zebrack BJ, Mills J, Weitzman TS. Health and supportive care needs of young adult cancer patients and survivors. J Cancer Surviv. 2007;1:137-45.

24. Kent EE, Smith AW, Keegan TH, et al. Talking about cancer and meeting peer survivors: Social information needs of adolescents and young adults diagnosed with cancer. J Adolesc Young Adult Oncol. 2013;2:44-52.

25. Osborn M, Little C, Bowering S, et al. Youth Cancer Services in Australia: Development and implementation. International perspectives on AYAO, Part 3. J Adolesc Young Adult Oncol. 2013;2:118-24.

26. Anazodo A, Chard J. Medical and psychosocial challenges in caring for adolescent and young adult patients with cancer Cancer Forum. 2013;37(1):23-6.

27. Skaczkowski G, White V, Thompson K, et al. Factors influencing the provision of fertility counselling and impact on quality of life in Adolescents and Young Adults with cancer. Submitted manuscript.

28. Carey M, Leropoli S, White V. The PROSPECT program. Patient responses: An ongoing survey of people experiencing cancer treatment. Pilot Study. Melbourne, Australia: Centre for Behavioural Research in Cancer, The Cancer Council Victoria, Prepared for: CanNET Victoria; 2009 August.

29. Ferrari A, Thomas D, Franklin AR, et al. Starting an adolescent and young adult program: some success stories and some obstacles to overcome. J Clin Oncol. 2010;28:4850-7.

30. Oberoi DV, White V, Jefford M, et al. Caregivers' information needs and their 'experiences of care' during treatment are associated with elevated anxiety and depression: A cross-sectional study of the caregivers of renal cancer survivors. Support Care Cancer. 2016;24:4177-86.

31. Cella DF, Tulsky DS, Gray G, et al. The Functional Assessment of Cancer Therapy scale: Development and validation of the general measure. J Clin Oncol. 1993;11:570-9. 
32. Janda M, DiSipio T, Hurst C, et al. The Queensland cancer risk study: General population norms for the Functional Assessment of Cancer Therapy-General (FACTG). Psycho-Oncology. 2009;18:606-14.

33. Psycho-oncology Co-operative Research Group. Calculating norm-based T-scores from your FACT-G data - spss syntax. 2009 [8 June 2016]; Available from: http://www.pocog.org.au/doc/FACT_norm_syntax_complete.txt.

34. Hosmer DW, Lemeshow S. Applied Logistic Regression. New York: Wiley; 2000.

35. White V, Skaczkowski G, Thompson K, et al. Experiences of care of Adolescents and Young Adults (AYAs) with cancer in Australia. submitted.

36. Kleeberg UR, Tews JT, Ruprecht $T$, et al. Patient satisfaction and quality of life in cancer outpatients: results of the PASQOC study. Support Care Cancer. 2005;13(5):303-10.

37. Maurice-Stam H, Grootenhuis M, Brons $P$, et al. Psychosocial indicators of healthrelated quality of life in children with cancer 2 months after end of successful treatment. J Pediatr Hematol Oncol. 2007;29:540-50.

38. Sung L, Klaassen R, Dix D, et al. Identification of paediatric cancer patients with poor quality of life. Br J Cancer. 2009;100:82-8.

39. Fernandez C, Fraser GA, Freeman C, et al. Principles and recommendations for the provision of healthcare in Canada to adolescent and young adult-aged cancer patients and survivors. J Adolesc Young Adult Oncol. 2011;1:53-9.

40. National Comprehensive Cancer Network. Adolescent and Young Adult (AYA) Oncology. 2012.

41. National Institute for Health and Clinical Excellence (NICE). Cancer services for children and young people. Quality standard (QS55). London, UK: NICE, 2014.

42. Smith S, Case L, Waterhouse K. A blueprint of care for teenagers and young adults with cancer. London, UK: Teenage Cancer Trust, 2012.

43. Korsvold L, Mellblom AV, Lie HC, et al. Patient-provider communication about the emotional cues and concerns of adolescent and young adult patients and their family members when receiving a diagnosis of cancer. Patient Educ Couns. 2016;99:1576-83

44. Wilkes S, Coulson S, Crosland A, et al. Experience of fertility preservation among younger people diagnosed with cancer. Hum Fertil. 2010;13:151-8.

45. McCarthy MC, McNeil R, Drew S, et al. Psychological distress and posttraumatic stress symptoms in adolescents and young adults with cancer and their parents. J Adolesc Young Adult Oncol. 2016;5(4):322-9. 
Table 1. Relationship between experiences of care and quality of life

\begin{tabular}{|c|c|c|c|c|c|c|c|c|c|c|c|c|c|c|c|}
\hline \multirow[b]{3}{*}{$\begin{array}{l}\text { Communication/Support } \\
(\mathrm{n}=204)\end{array}$} & \multicolumn{3}{|c|}{$\begin{array}{c}\text { PWB } \\
\mathrm{M}(\mathrm{SE})=22.77(.40)\end{array}$} & \multicolumn{3}{|c|}{$\begin{array}{c}\text { SWB } \\
\mathrm{M}(\mathrm{SE})=\mathbf{2 2 . 2 8}(.36) \\
\end{array}$} & \multicolumn{3}{|c|}{$\begin{array}{c}\text { FWB } \\
M(\text { SE })=20.92(.45) \\
\end{array}$} & \multicolumn{3}{|c|}{$\begin{array}{c}\text { EWB } \\
\mathrm{M}(\mathrm{SE})=18.81(.29)\end{array}$} & \multicolumn{3}{|c|}{$\begin{array}{c}\text { FACT-G } \\
\mathrm{M}(\mathrm{SE})=\mathbf{8 4 . 7 7 ( 1 . 1 5 )} \\
\end{array}$} \\
\hline & $\mathrm{F}$ & $\mathrm{B}(\mathrm{SE})$ & $p$ & $\mathrm{~F}$ & $\mathrm{~B}(\mathrm{SE})$ & $p$ & $\mathrm{~F}$ & $\mathrm{~B}(\mathrm{SE})$ & $p$ & $\mathrm{~F}$ & $\mathrm{~B}(\mathrm{SE})$ & $p$ & $\mathrm{~F}$ & $\mathrm{~B}(\mathrm{SE})$ & $p$ \\
\hline & 3.51 & & $<.001$ & 3.88 & & $<.001$ & 4.38 & & $<.001$ & 3.91 & & $<.001$ & 5.24 & & $<.001$ \\
\hline Communication/Support & & $3.14(1.21)$ & .010 & & $5.88(1.07)$ & $<.001$ & & $5.03(1.32)$ & $<.001$ & & $2.55(0.87)$ & .004 & & $16.72(3.34)$ & $<.001$ \\
\hline \multicolumn{16}{|l|}{ Diagnosis $(n=191)$} \\
\hline Step 1 & 2.45 & & .009 & 2.67 & & .005 & 3.33 & & .001 & 3.43 & & $<.001$ & 3.37 & & $<.001$ \\
\hline Diagnosis & & $.85(1.42)$ & .550 & & $5.58(1.27)$ & $<.001$ & & $4.21(1.56)$ & .008 & & $2.56(1.01)$ & .012 & & $13.30(4.00)$ & .001 \\
\hline Step 2 & 3.20 & & .001 & 3.53 & & $<.001$ & 3.86 & & $<.001$ & 3.52 & & $<.001$ & 4.61 & & $<.001$ \\
\hline Diagnosis & & $-2.58(1.77)$ & .147 & & $2.38(1.57)$ & .132 & & $.80(1.96)$ & .681 & & $.99(1.28)$ & .441 & & $1.67(4.92)$ & .735 \\
\hline Communication/Support & & $4.97(1.61)$ & .002 & & $4.67(1.43)$ & .001 & & $4.98(1.78)$ & .006 & & $2.30(1.16)$ & .049 & & $17.01(4.46)$ & $<.001$ \\
\hline \multicolumn{16}{|l|}{$\begin{array}{l}\text { Treatment experiences } \\
(\mathbf{n = 2 0 3})\end{array}$} \\
\hline Step 1 & 3.02 & & .001 & 3.87 & & $<.001$ & 4.04 & & $<.001$ & 4.01 & & $<.001$ & 4.69 & & $<.001$ \\
\hline Treatment experiences & & $2.00(1.25)$ & .111 & & $5.90(1.10)$ & $<.001$ & & $4.57(1.36)$ & .001 & & $2.81(0.89)$ & .002 & & $15.34(3.44)$ & $<.001$ \\
\hline Step 2 & 3.25 & & $<.001$ & 4.20 & & $<.001$ & 4.15 & & $<.001$ & 3.75 & & $<.001$ & 5.11 & & $<.001$ \\
\hline Treatment experiences & & $-.95(1.82)$ & .602 & & $2.97(1.58)$ & .062 & & $1.51(1.97)$ & .445 & & $1.79(1.30)$ & .169 & & $5.33(4.95)$ & .283 \\
\hline Communication/Support & & $3.95(1.78)$ & .028 & & $3.94(1.55)$ & .012 & & $4.12(1.94)$ & .035 & & $1.37(1.28)$ & .286 & & $13.48(4.87)$ & .006 \\
\hline \multicolumn{16}{|l|}{$\begin{array}{l}\text { Inpatient experiences } \\
(\mathrm{n}=140)\end{array}$} \\
\hline Step 1 & 2.85 & & .003 & 2.05 & & .034 & 3.54 & & $<.001$ & 3.61 & & $<.001$ & 3.80 & & $<.001$ \\
\hline Inpatient experiences & & $3.44(1.39)$ & .014 & & $3.60(1.17)$ & .003 & & $5.15(1.46)$ & .001 & & $3.59(0.96)$ & $<.001$ & & $15.91(3.80)$ & $<.001$ \\
\hline Step 2 & 2.83 & & .002 & 2.47 & & .008 & 3.56 & & $<.001$ & 3.29 & & .001 & 3.99 & & $<.001$ \\
\hline Inpatient experiences & & $1.77(1.76)$ & .315 & & $1.42(1.45)$ & .331 & & $3.14(1.84)$ & .090 & & $3.18(1.22)$ & .010 & & $9.53(4.75)$ & .047 \\
\hline Communication/Support & & $2.94(1.91)$ & .127 & & $3.88(1.59)$ & .016 & & $3.58(2.01)$ & .077 & & $.72(1.34)$ & .593 & & $11.36(5.21)$ & .031 \\
\hline
\end{tabular}

This article is protected by copyright. All rights reserved. 
Step 1

Step 2

\begin{tabular}{|c|c|c|c|c|c|c|c|c|c|c|c|c|c|c|c|}
\hline & 1.69 & & .088 & 2.52 & & .008 & 3.34 & & .001 & 3.11 & & .001 & 3.57 & & $<.001$ \\
\hline Finishing treatment & & $2.39(1.33)$ & .074 & & $5.49(1.35)$ & $<.001$ & & $5.47(1.56)$ & .001 & & $2.39(1.20)$ & .031 & & $15.75(4.05)$ & $<.001$ \\
\hline 2 & 1.58 & & .110 & 3.48 & & $<.001$ & 3.27 & & .001 & 3.17 & & .001 & 3.89 & & $<.001$ \\
\hline $\mathrm{Fi}$ & & 1.7 & .395 & & $1.54(1.75)$ & .380 & & $3.34(2.09)$ & .112 & & .61 & .675 & & 6. & 195 \\
\hline Communication/Support & & $1.24(1.72)$ & .471 & & $5.72(1.70)$ & .001 & & $3.09(2.03)$ & .130 & & $2.57(1.42)$ & .072 & & $12.70(5.20)$ & 016 \\
\hline
\end{tabular}

Note: Control variables in each model were: sex, diagnosis, remoteness of residence and time since diagnosis. $\mathrm{PWB}=$ physical well-being, SWB=social well-being,

FWB=functional well-being, EWB=emotional well-being, FACT-G=overall well-being. Due to missing data, sample sizes for SWB, FWB, EWB and FACT-G analyses

were: Communication/Support $n=203$, Diagnosis $n=190$, Treatment experiences $n=202$, Inpatient experiences $n=139$, Finishing treatment $n=156$.

Significant results are bolded. 
Table 2. Correlation $(r)$ between EOC scales and mean subscale scores

\begin{tabular}{lcccccr}
\hline Range for all scales=0-1 & Mean(SD) & 1 & 2 & 3 & 4 & 5 \\
\hline (1)Diagnosis & $.41(.31)$ & & & & & \\
(2)Overall treatment & $.63(.32)$ & $.47^{* * *}$ & & & & \\
(3)Inpatient experiences & $.62(.36)$ & $.35^{* * *}$ & $.67 * * *$ & & & \\
(4)Finishing treatment & $.31(.30)$ & $.44 * * *$ & $.56^{* * *}$ & $.48^{* * *}$ & & \\
(5)Communication/Support & $.48(.34)$ & $.65^{* * *}$ & $.73^{* * *}$ & $.62^{* * *}$ & $.67 * * *$ & \\
\hline
\end{tabular}

$* * * p<.001$ 


\section{University Library}

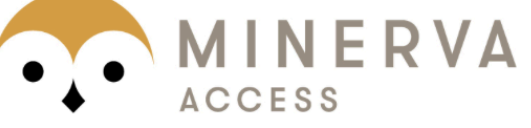

A gateway to Melbourne's research publications

Minerva Access is the Institutional Repository of The University of Melbourne

Author/s:

Skaczkowski, G;White, V;Thompson, K;Bibby, H;Coory, M;Pinkerton, R;Orme, LM;Conyers, R;Phillips, MB;Osborn, M;Harrup, R;Anazodo, A

Title:

Do Australian adolescents' and young adults' experiences of cancer care influence their quality of life?

Date:

2018-03-01

Citation:

Skaczkowski, G., White, V., Thompson, K., Bibby, H., Coory, M., Pinkerton, R., Orme, L. M., Conyers, R., Phillips, M. B., Osborn, M., Harrup, R. \& Anazodo, A. (2018). Do Australian adolescents' and young adults' experiences of cancer care influence their quality of life?. PSYCHO-ONCOLOGY, 27 (3), pp.990-997. https://doi.org/10.1002/pon.4625.

Persistent Link:

http://hdl.handle.net/11343/283460 